\title{
CERRAMIENTO DE VIDRIO PARA UN EDIFICIO. POLIDEPORTIVO EN MADRID/ESPAÑA
}

\author{
(GLASS WALL FOR A SPORTS COMPLEX IN MADRID, SPAIN)
}

Salvador Pérez Arroyo y Eva Hurtado Torán, Arquitectos.

\section{RESUMEN}

Presentamos un análisis del cerramiento de vidrio realizado en un edificio polideportivo en Madrid, en el que el deporte se organiza en altura, en una construcción de cinco plantas, una de cuyas fachadas se concibe como totalmente

transparente, mostrando el edificio "seccionado" al exterior.

El muro cortina, de $47,11 \times 20,24 \mathrm{~m}$ de superficie, se resuelve con grandes piezas de vidrio laminado, de 2,20 ×3,00 m, aproximadamente, sujetas mediante seis piezas de presión a la estructura metálica que cuelga enteramente de la cubierta. Aparece este material al exterior sin carpintería, con juntas abiertas que funcionan como canales de drenaje.

Hoy el desarrollo de la construcción y la tecnología hacen posible el planteamiento de cerramientos cuya flexibilidad de funcionamiento se adapta a las condiciones exteriores y proporciona mayores prestaciones que los de construcción tradicional. Ello es especialmente importante en edificios de uso público que, como éste, tienen que aparecer en la ciudad con una imagen singular y estimulante, mostrando a veces, como en este caso, su actividad hacia la calle.

\section{SUMMARY}

This is an analysis of a glass wall realized in a sports centre in Madrid, in which sport takes place above ground level, in a building of five storeys. The façade of one storey is designed to be totally transparent, so that the building from the outside gives the impression of being ain sections".

The window-wall, with a surface area of approximately $47.11 \mathrm{~m}$ by $20.24 \mathrm{~m}$, is achieved by using large sheets of laminated glass, approximately $2.20 \mathrm{~m}$ by $3.00 \mathrm{~m}$, held in place by six suction devices in a metal frame suspended without other support from the roof. From the exterior this material appears with no visible carpintery, with open joints which act as drainage channels.

With recent developments in building and technology we can consider using wall materials adaptable to all external conditions, and which allow more possibilities than traditional construction materials. This is especially important for public amenity buildings which, like this one, must present an original and stimulating image, at times, as in this case, displaying to the outside world the activity within.

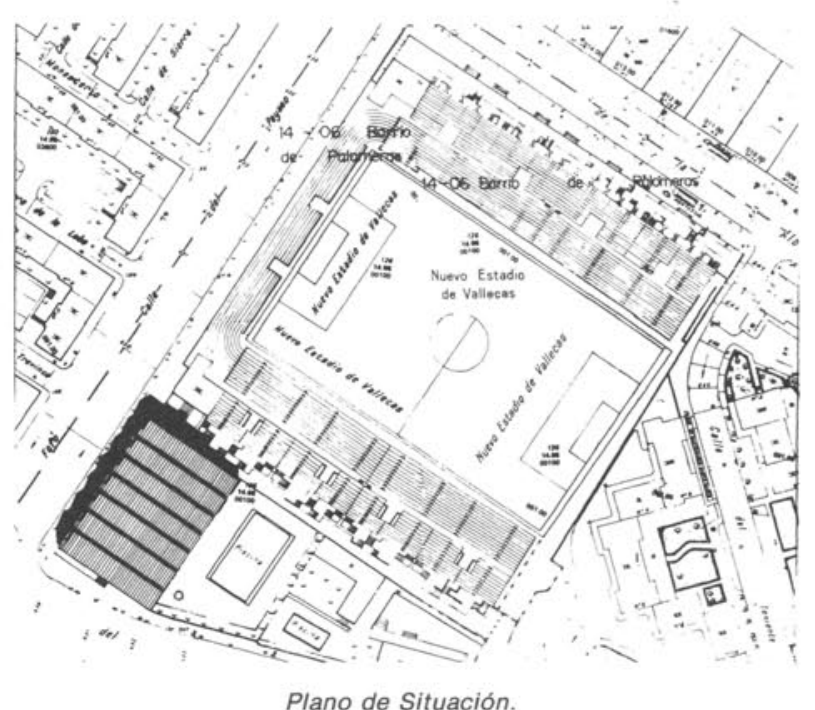

Plano de Situación.
En el año 1922 Adolf Behne publicó un artículo en la revista Frülicht titulado "Arquitectos" en el que hacia una referencia a la arquitectura de J. J. P. Oud en los siguientes términos:

"Su meta, es decir una arquitectura positiva basada sobre las exigencias de la vida moderna y realizada con una dedicación total, casi despersonalizada, libre de las inclinaciones y de los humores del creador, una arquitectura apoyada en todas las nuevas conquistas de la técnica, que da forma a los organismos animados del espiritu, racionalmente estudiados, que viven en la relación pura e intangible de las distintas partes entre ellas y con el conjunto, sin ir más a la busca de ningún interesante juego de elementos accesorios contrastantes." 


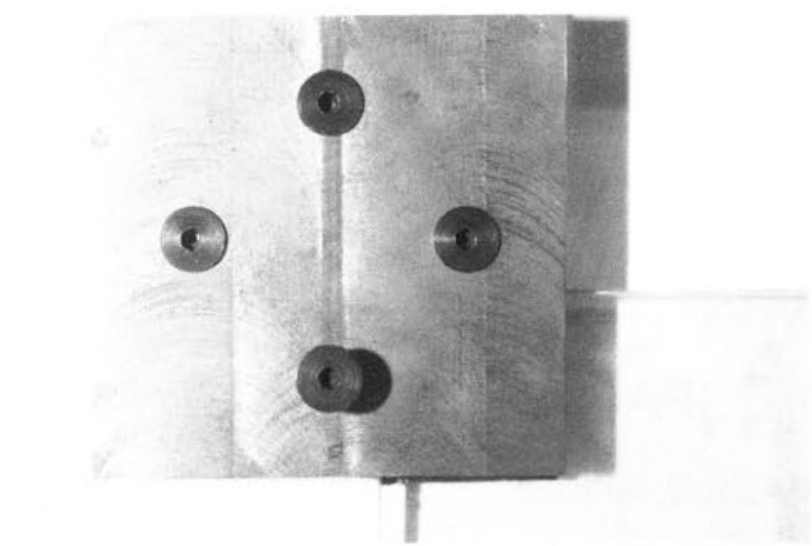

Modelo de la primera solución de elementos de sujeción del vidrio, por presión en las esquinas de las piezas.

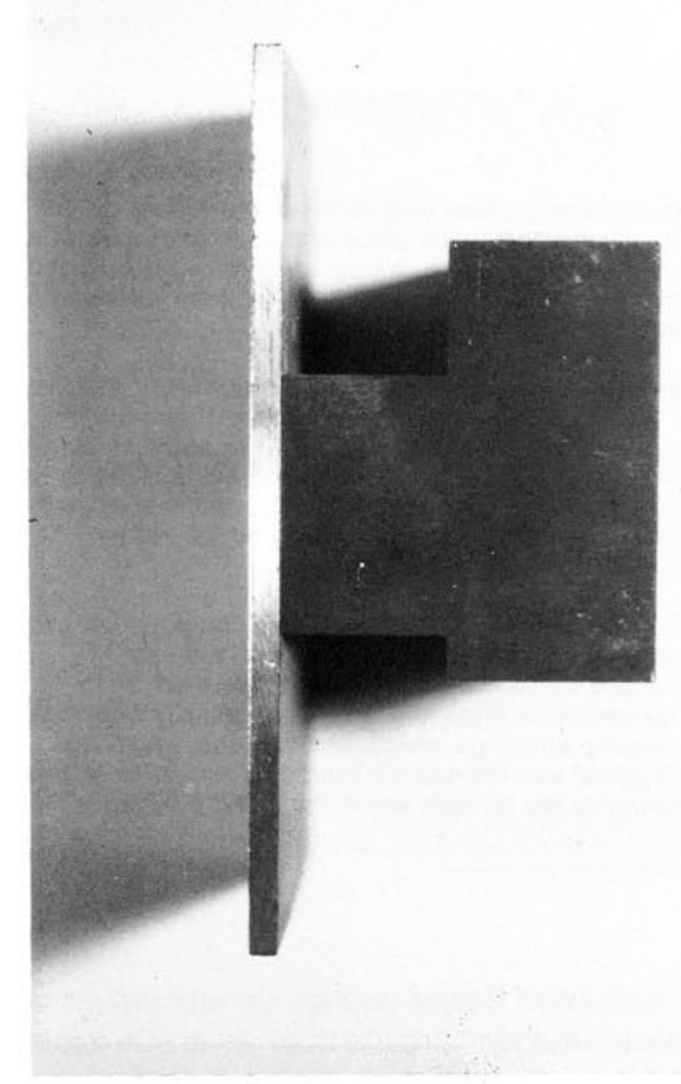

Solución definitiva de elemento de sujeción del vidrio.

Las ideas expuestas por Behne pretendian hacer una crítica de las tendencias expresionistas del momento, particularmente de la obra de Pöelzig. Hoy nuestro in terés está en buscar una arquitectura que utilice al máximo las posibilidades que la técnica actual puede proporcionar. La cualificación de la arquitectura contem. poránea en términos acordes con la cultura de la so. ciedad en la que vivimos.

En este artículo pretendemos reflexionar sobre los ce(C) Consejo Superior de Investigaciones Científicas Licencia Creative Commons 3.0 España (by-nc)

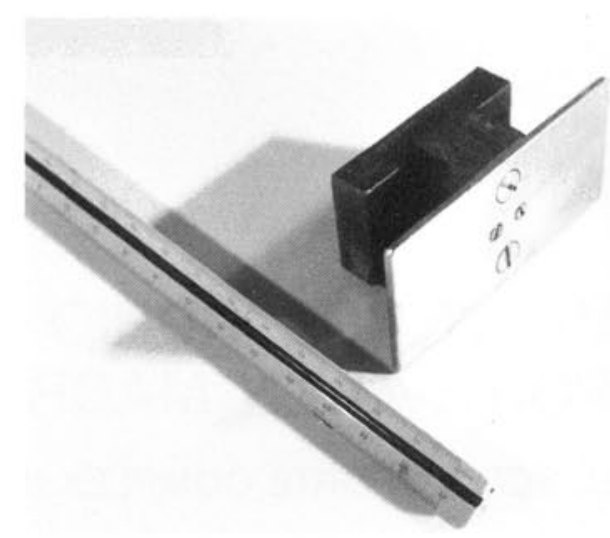

Perfil de solución intermedia de elemento de sujeción del vidrio, con escudo de acero inoxidable y pieza martillo cuya forma permite el paso del agua por la parte inferior del canal.

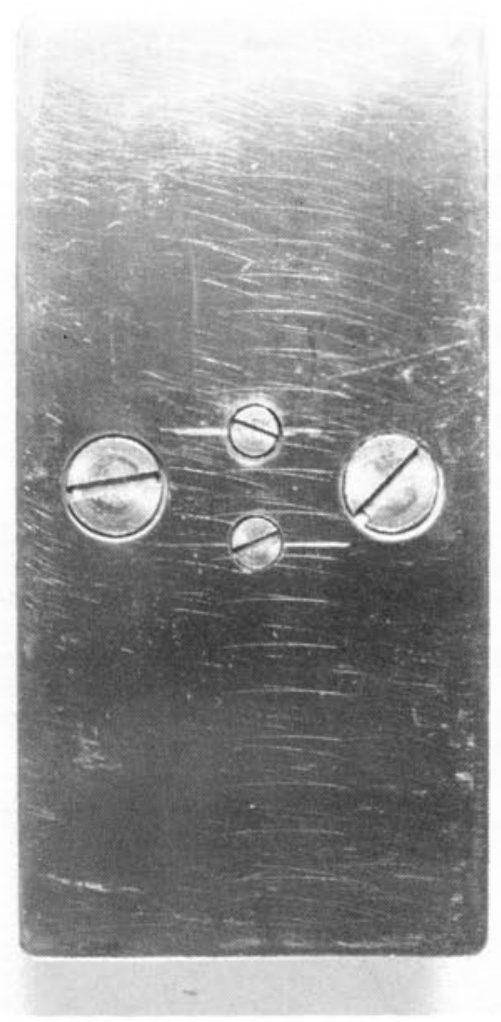

Escudo de acero inoxidable de presión sobre el vidrio. Solución definitiva.

rramientos de vidrio sirviéndonos de un ejemplo, el realizado en un edificio polideportivo de Vallecas. Un espacio de singulares características, por la concentración de funciones y por el planteamiento y la integración de las mismas.

La lógica que ha presidido el proyecto ha sido la de proporcionar un amplio número de servicios en un solar muy reducido y poner en contacto estos espacios con el exterior cuando fuera posible. Para conseguirlo se http://informesdelaconstruccion.revistas.csic.es 
ha pensado en una fachada de vidrio que hace posible, desde la calle, ver la intensa actividad deportiva del centro, estimulando a los que desde el exterior se paren a observar. Una cubierta móvil que permite en verano sentir que se está jugando al aire libre, libera la ganancia solar, que no se piensa compensar con aire frío artificial, fomentando por el contrario en este caso la ventilación natural, formando un muro Trombe.

Del mismo modo se ha previsto un vaso de piscina elevado que se conecta con unas piscinas exteriores por medio de un cerramiento, también móvil, que abre toda la fachada del lado opuesto.

El polideportivo se adosa al estadio de El Rayo Vallecano, dejando una calle interior peatonal entre ambos, y a la piscina descubierta existente. Quedan dos fachadas dando a las calles Payaso Fofó y Arroyo del Olivar. Las cuatro encierran el volumen prismático con que se ha concebido el edificio.

La complejidad del programa, la racionalidad y economía de uso y mantenimiento y las características del emplazamiento, condujeron desde los momentos iniciales al planteamiento de un polideportivo en altura, como la mejor manera de acometer el problema.

El edificio se concibe como un contenedor múltiple. que compartimenta el espacio interior, con cierta independencia del volumen exterior resultante, según las necesidades de uso. Asi los locales de servicio, vestuarios, aseos, guardarropas, oficinas..., se sitúan en una banda paralela a la fachada más larga que da a la calle Payaso Fofó, en cuatro plantas de alturas convencionales. La misma altura es ocupada, hacia la fachada opuesta, por el gran espacio de las piscinas cubiertas.

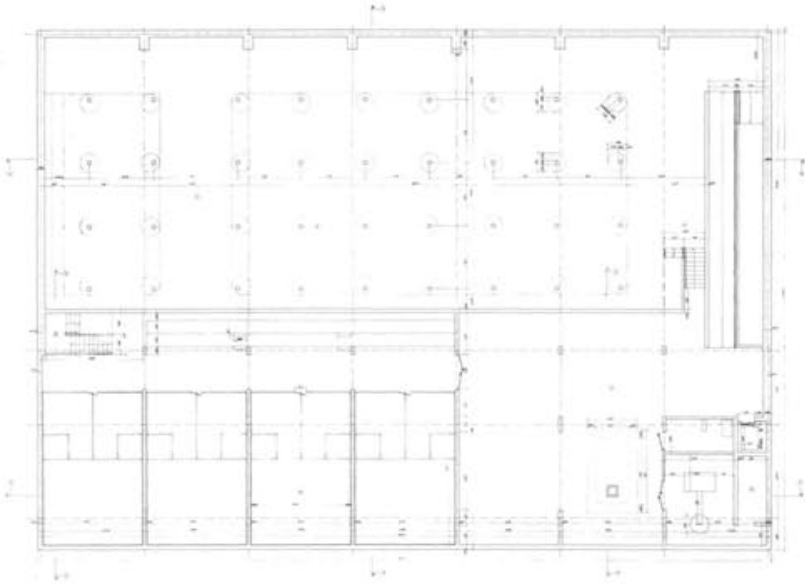

Planta Sótano: canchas de squash, oficinas e instalaciones.

(C) Consejo Superior de Investigaciones Científicas

Licencia Creative Commons 3.0 España (by-nc)
Interiormente este edificio se asienta sobre una planta sótano que además de cuatro canchas de squash, contiene locales de instalaciones.

En el nivel superior un espacio polideportivo ocupa la casi totalidad de la planta.

La transición entre estos espacios de diferentes alturas se resuelve en general con las zonas de paso, de conexión vertical, rampas o escaleras, y de graderío. El vestíbulo atraviesa el interior, con un hueco de triple altura.

Existen además dos ámbitos de particular importancia en este análisis sobre la superficie de vidrio del edificio: la cafeteria a media altura sobre el espacio de la piscina, como mirador hacia ella y la palestra de gimnasia que, entre plantas de servicios ciegas a la calle, se muestra, acristalada en sus dos frentes principales, al interior y al exterior.

En una edificación como la descrita, que funciona casi ininterrumpidamente a lo largo del dia y del año, es especialmente importante el tratamiento de las "pieles", para obtener un máximo aprovechamiento en los espacios iluminados naturalmente, soleados o sombreados, ventilados o herméticamente cerrados, según los requerimientos del momento.

De este modo, se cierra el edificio en tres de sus fachadas con superficies opacas de chapa metálica, salvo el frente de puertas plegables de piscina antes mencionado y algunos huecos laterales. Resta entonces la solución de la cuarta fachada que, por ser la que da entrada y muestra el polideportivo a la calle principal y por las características de lo que tras ella se organiza, es claramente diferente de las demás y por tanto de tratamiento singular.

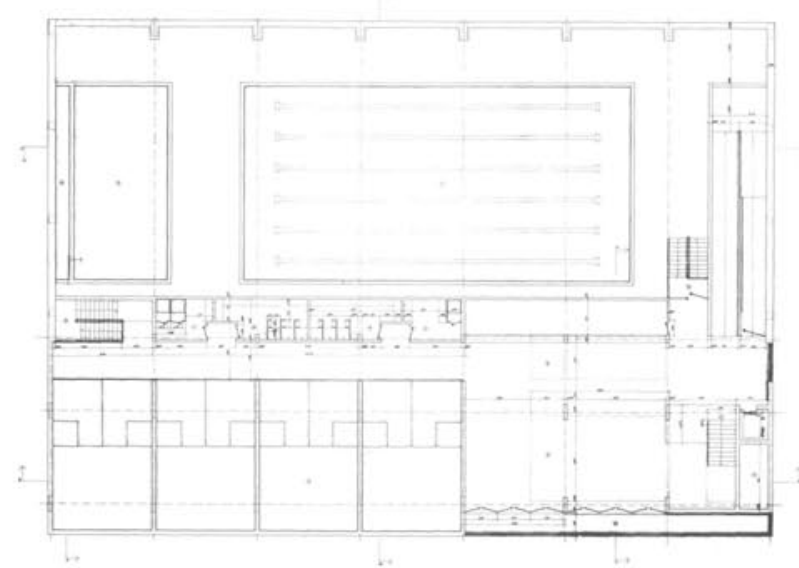

Planta Baja: vestibulo, vestuarios de squash y oficinas. 


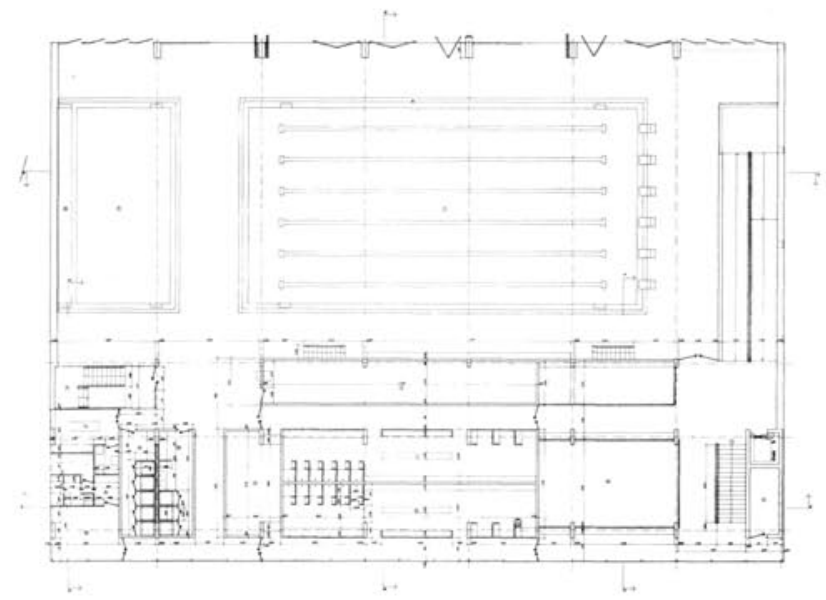

Planta Primera: vestuarios de piscina, piscinas cubiertas y locales mo. nitores.

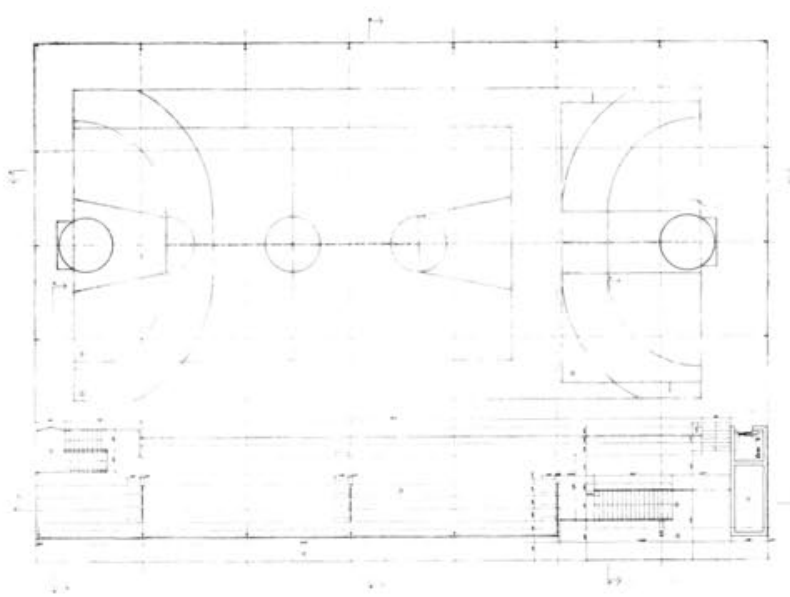

Planta Cuarta: polideportivo.

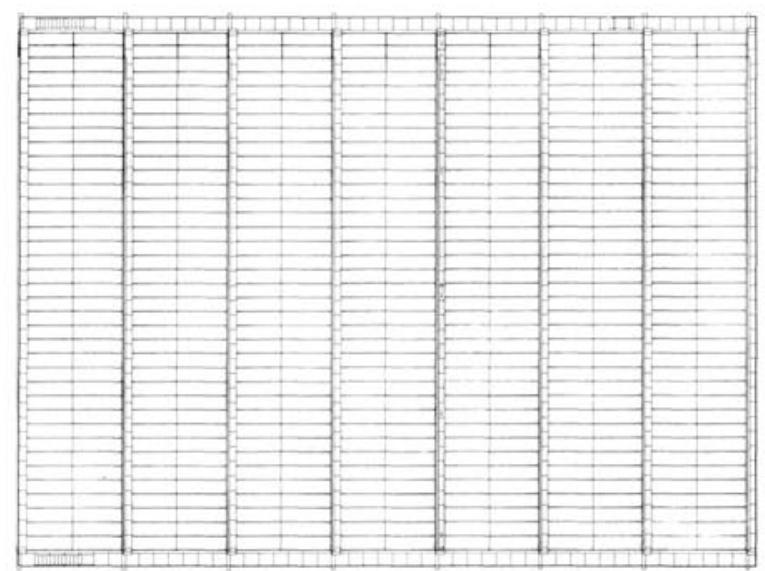

Planta de Cubiertas.

Se optó por hacerla totalmente transparente, con un muro cortina de grandes piezas y elementos de anclaje y carpintería reducidos al mínimo imprescindible, de modo que una lámina, que casi parece no existir, hiciera participar a la calle de las actividades del polideportivo, más en este caso, que abrirse al paisaje desde el interior.

Huyendo de todo expresionismo, tecnológico o formal, como se pone de manifiesto en el planteamiento del proyecto en su conjunto, también aqui se ha tratado de dar solución al problema con la mayor economía; en este caso el presupuesto era realmente escaso, y con la mayor sinceridad puesto que la lámina de vidrio no pretende ser más que lo que realmente es: un elemento superpuesto, de carácter específico, que como tal http://informesdelaconstruccion.revistas.csic.es 


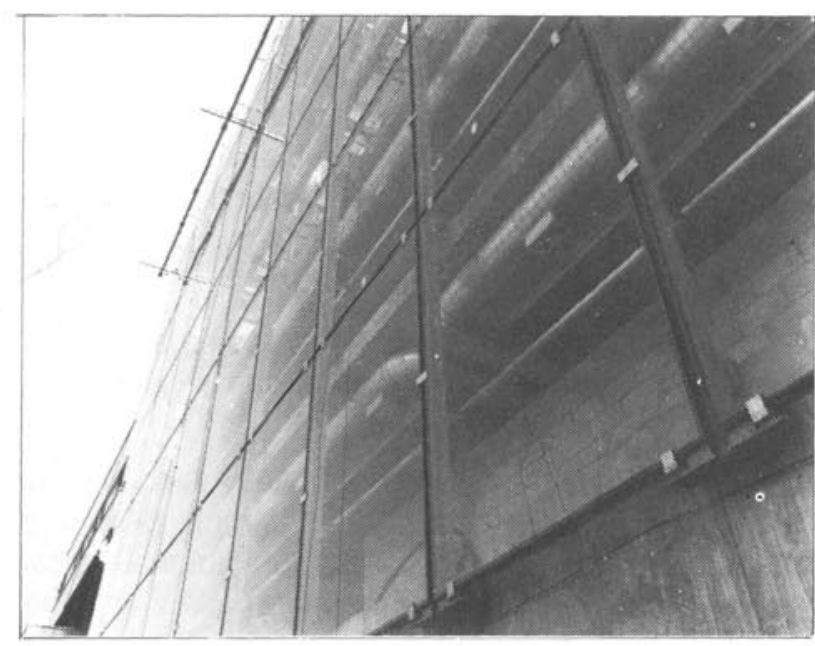

Detalle de piezas del cerramiento de vidrio.

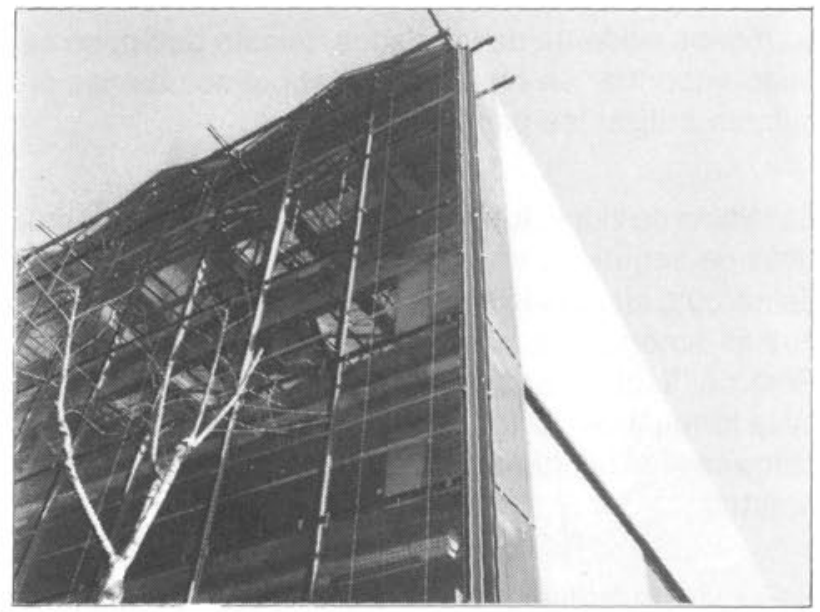

Detalle de solución de esquina.

se adhiere a la edificación mediante encuentros puntuales mínimos.

Dadas las condiciones de partida, escasez de soluciones de cierta calidad tecnológica que ofrece el mercado nacional, construcción de bajo coste, plazos de obra y necesidades de seguridad y acondicionamiento, ha sido necesario un acelerado proceso, de búsqueda, prueba y error, en el que han participado técnicos y fabricantes de diversos oficios.

El frente tiene una longitud de $47,11 \mathrm{~m}$ y una altura total de 20,24 m en su punto más alto. Un muro de hormigón visto de $3 \mathrm{~m}$ recorre la fachada a modo de basamento en continuidad con los de mayor altura de las laterales, y desaparece para dar lugar a la entrada del

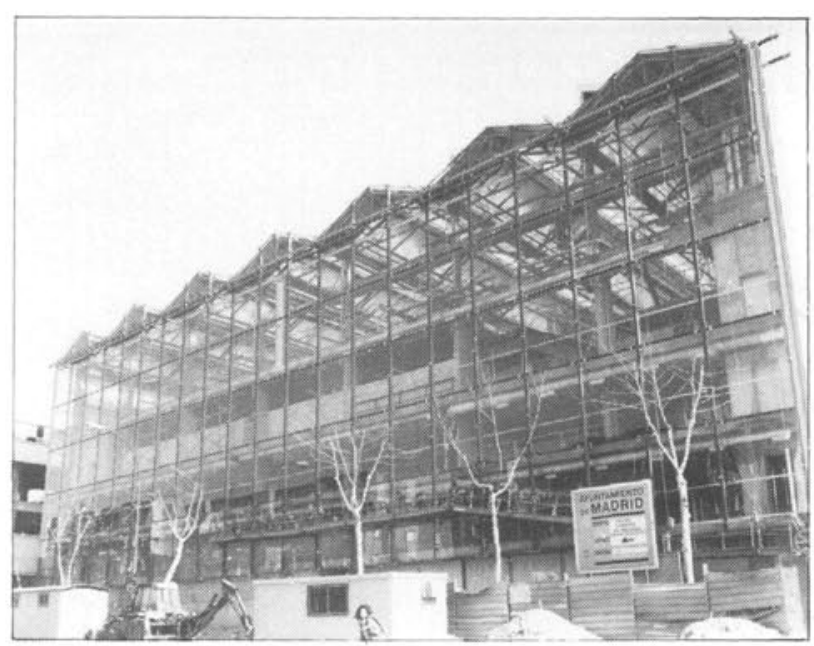

Alzado del muro cortina.

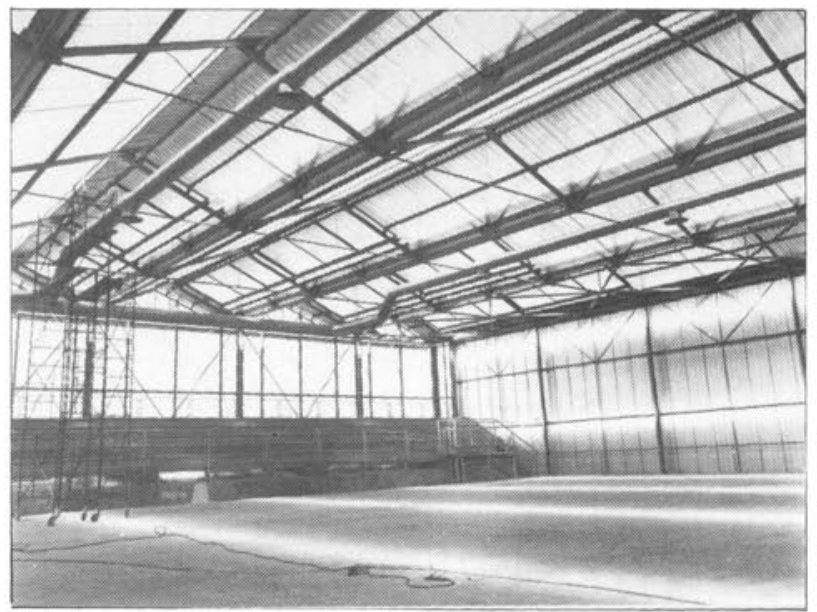

Interior del polideportivo hacia muro de vidrio.

edificio, único retranqueo que rompe el volumen puro en todo el perimetro.

Sobre este zócalo, el plano de vidrio con una altura por tanto de 17,24 m abraza los muros laterales manifestando al exterior la sección del edificio.

La estructura principal se organiza en siete módulos de $6,68 \mathrm{~m}$, lo que se acusa en el muro de vidrio con piezas al tercio de esta dimensión.

Unos perfiles IPE 120 soldados a la viga triangulada de cubierta, de 16,59 m de longitud forman la estructura primaria del muro. Sobre ellos, la cuadrícula constituye la base de apoyo de las piezas de vidrio de $3.004 \mathrm{~m}$, en vertical por $2.227 \mathrm{~m}$ en horizontal, con montantes 


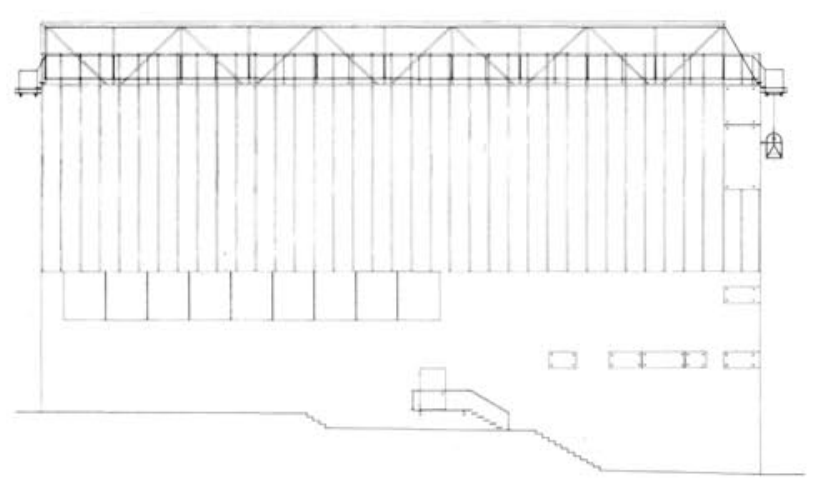

Alzado N.E.

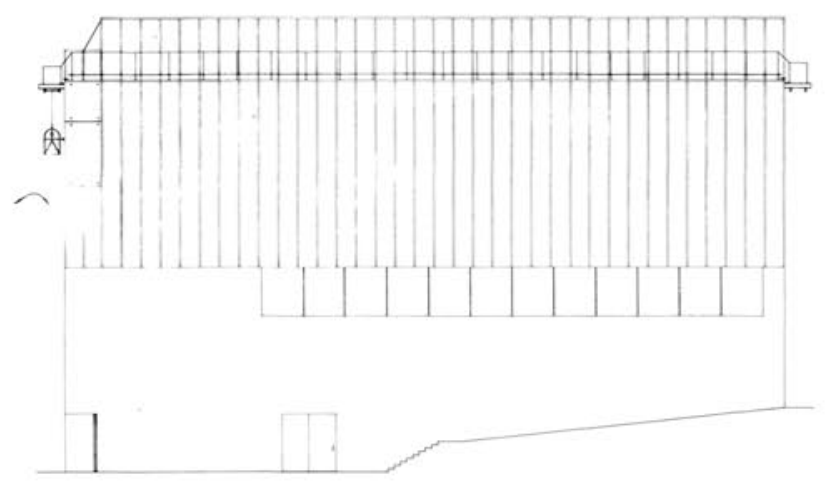

Alzado S.O.

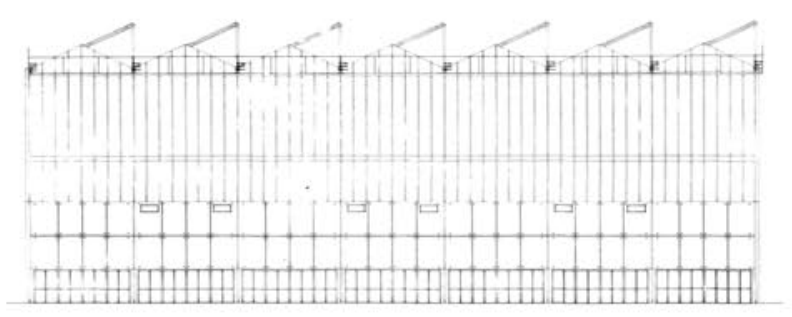

Alzado S.E.

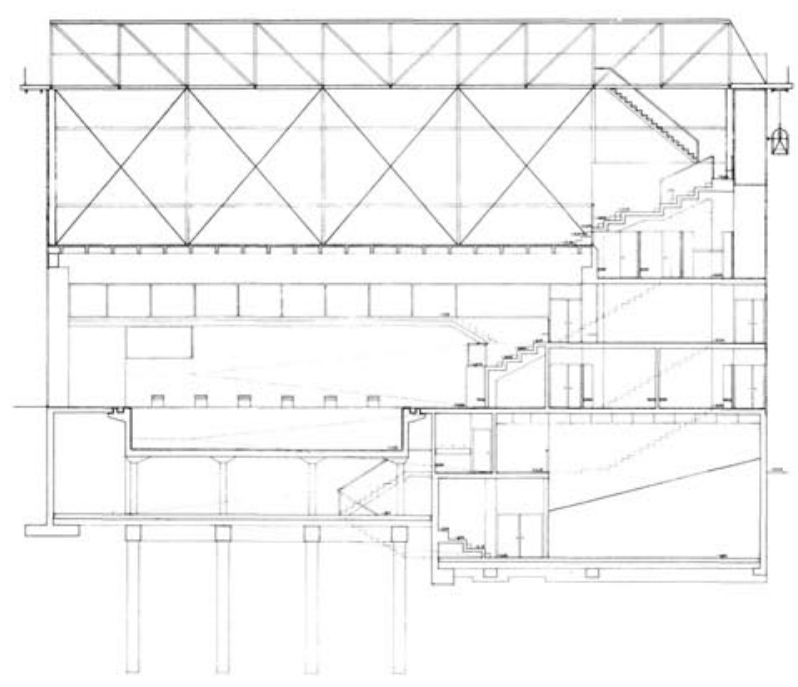

Sección transversal (por piscina).

(C) Consejo Superior de Investigaciones Científicas Licencia Creative Commons 3.0 España (by-nc) $y$ travesaños formados por perfiles abiertos en forma de $C$ de $100 \times 38 \mathrm{~mm}$, boca de $65 \mathrm{~mm}$ y 6 de pared. Los montantes se sueldan a los IPE anteriores y los travesaños a ellos, a modo de brazos que se encuentran en el centro de cada módulo con un pequeño caballete de junta.

Sobre la trama descrita, con dos juntas de dilatación en toda su longitud, se fijan las piezas de vidrio.

Resuelta la sujeción del muro cortina, y dado que las características del mismo lo permitian (no tiene que abrirse ni necesita ningún elemento adicional de parasol), se pensó en vidrio sin carpintería. Así el plano aparece libre de divisiones de otros materiales, con juntas abiertas de 52 y $51,6 \mathrm{~mm}$, que como delgadas hendiduras de sombra ordenan imperceptiblemente la superficie.

La elección del modo de sujeción del vidrio primero, y de su solución concreta después, resultó ser el paso menos evidente de los dados, puesto que poco se pudo encontrar en nuestro pais sobre soluciones similares utilizables para este caso.

La lámina de vidrio, totalmente transparente, es por motivos de seguridad un laminar de $6+6 \mathrm{~mm}$, resultado del cálculo efectuado según lo especificado en los manuales americanos y, particularmente, en el libro de Rostron "Light Cladding of Buildings" donde se aplica la fórmula del factor " $R$ ", con un cuadro que lo relaciona con las condiciones exteriores y las de apoyo perimetral.

No existe un manual apto para este tipo de exigencias verdaderamente actualizado, lo que nos parece puede dificultar en estos casos la labor de elección del arquitecto.

En el muro que analizamos, y como se puede apreciar en los detalles, cada pieza de vidrio se sujeta en 6 puntos con unos escudos de acero inoxidable de $140 \times 70$ milímetros y 4 de espesor, que presionan sobre la lámina de vidrio a través de elementos de neopreno que, en bandas continuas de 10 ó $15 \mathrm{~mm}$ de espesor, mantienen la pieza apoyada sobre un marco elástico para absorber todas las tensiones transmitidas desde el vidrio.

La tensión se transmite a la estructura de los canales, que se han reforzado en estos puntos, por medio de una pieza martillo de acero galvanizado de $65 \times 70 \times 20$ milímetros, solidaria al escudo exterior por dos parejas de tornillos. Estos se han fijado posteriormente con resina, en previsión de posibles aflojamientos por vibración.

http://informesdelaconstruccion.revistas.csic.es 

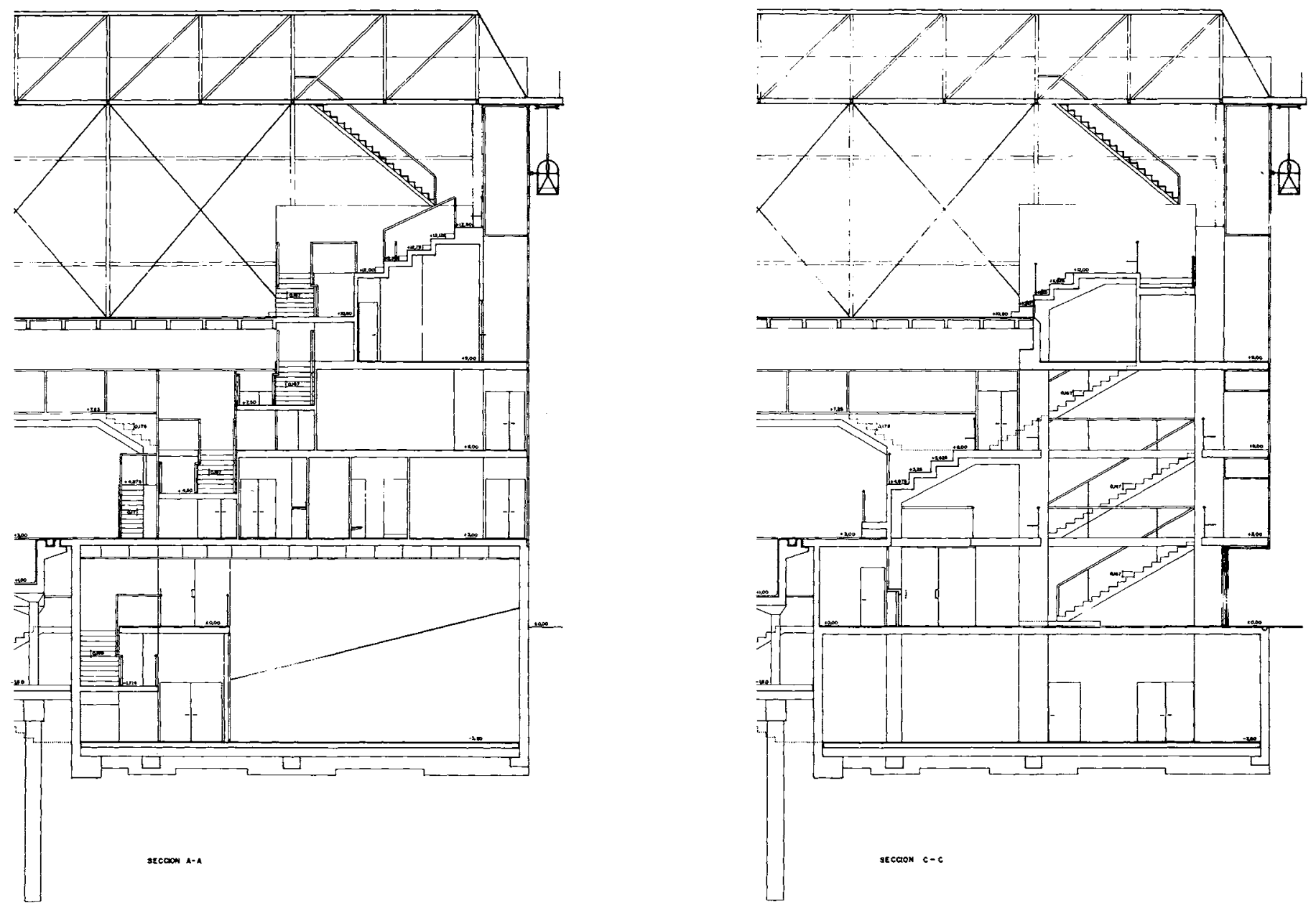

Secciones transversales (por squash y por vestíbulo).

Los elementos de la reja que soporta el muro son a su vez los canales de drenaje horizontales y verticales y carril para la góndola de limpieza. La resistencia a viento la proporciona la inercia de estos elementos y los forjados sobre los que se apoyan mediante angular $250 \times 100 \times 10$ y chapa $100 \times 6$, que absorben movimientos en el plano horizontal y vertical. Todo el muro está concebido como una "cortina" colgada de la cubierta, funcionando como tirantes los montantes verticales de la reja y los perfiles IPE 120 de refuerzo, añadidos a estos canales.

La orientación de la fachada es Noroeste, con una cierta exposición a los ponientes en verano y, por la edificación existente, prácticamente nula en invierno. Estas condiciones desfavorables quedan compensadas por la construcción del cerramiento como un muro Trombe, que ventila a través de la apertura mecánica de la cubierta y de las rendijas de ventilación de los forjados, de 21,5 y $26,5 \mathrm{~cm}$ de anchura, que son practicables para regular el tiro en función de la estación. Las instalaciones de calefacción por aire se dirigen también hacia el muro compensando efectos posibles de condensación.
Hay que recordar que la función estricta de este edificio exige unos planteamientos distintos en función del área tratada. La piscina requiere un tratamiento cuidadoso del calor y de la humedad. La cancha de baloncesto puede convertir en verano el espacio cerrado en uno semiabierto muy confortable.

La experiencia adquirida en la construcción del edificio nos habla de la necesidad de estudiar profundamente muchos de los conceptos tradicionales de ventilación y cierre de los espacios, recuperando la posibilidad de regular en estos edificios las aperturas y cierres de los mismos, aprovechando asi factores naturales de confort y permitiendo a la vez reconsiderar algunos conceptos de uso.

Los muros de vidrio han sido en la historia de la arquitectura reciente, desde el inicio del Movimiento Moderno, un símbolo de cambio. Muchos edificios incorporaron, desde el inicio, este tipo de soluciones con resultados muy diversos.

Le Corbusier inició de un modo intuitivo el planteamiento de los muros respirantes en los edificios del 
Ejército de Salvación en París y en el Pabellón Suizo de la Ciudad Universitaria. Hoy se encuentran ambos sustancialmente modificados. Otros ejemplos servirían para demostrar cómo este tipo de solución constructiva ha sido tratada con distinta suerte. Particularmente interesante es el caso del edificio construido para la Olivetti por Pollini y Figini en Ivrea, en 1939, o las obras de Gropius en Dessau y Alfeld.

La utilización masiva de los muros de vidrio desde los años cincuenta, en todos los edificios de oficinas y particularmente en las grandes edificaciones en altura, significa un paso adelante en el tratamiento de este tipo de problemas. El edificio Pirelli en Milán de G. Ponti o la Lever House de los S.O.M. en Nueva York son ejemplos brillantes.

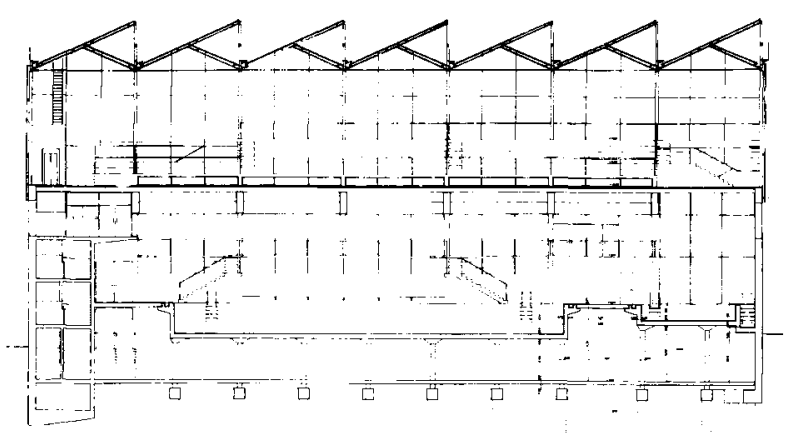

Sección longitudinal (por piscina).
En la actualidad el tratamiento de este tipo de cerramientos se une a un renacer de la vieja idea del edificio con una capacidad técnica importante. La definición de edificio inteligente nos habla del concepto constructivo de flexibilidad y adaptación a las distintas exigencias ambientales o de uso, frente a la inmutabilidad de la arquitectura tradicional.

En algunos casos estos edificios se conciben cargados de instalaciones, enfatizando la componente tecnológica, lo que es a todas luces erróneo. No es, por lo tanto, el valor de la técnica lo que prima o caracteriza este planteamiento, es la riqueza de respuestas la que hace a un edificio alejarse de la idea tradicional de lo construido.

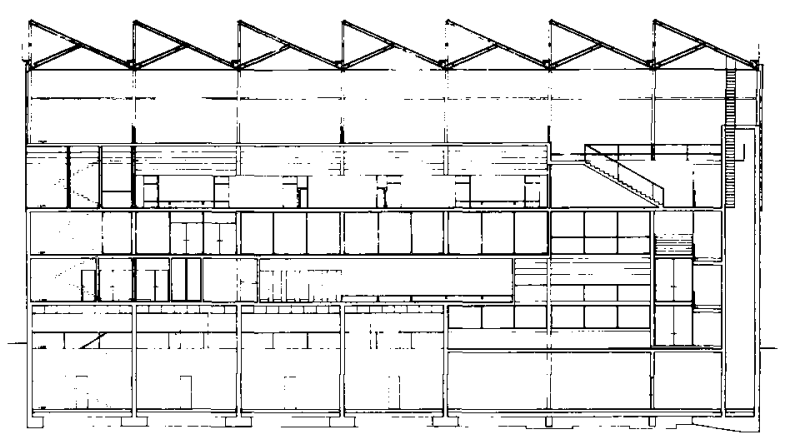

Sección longitudinal (por squash).

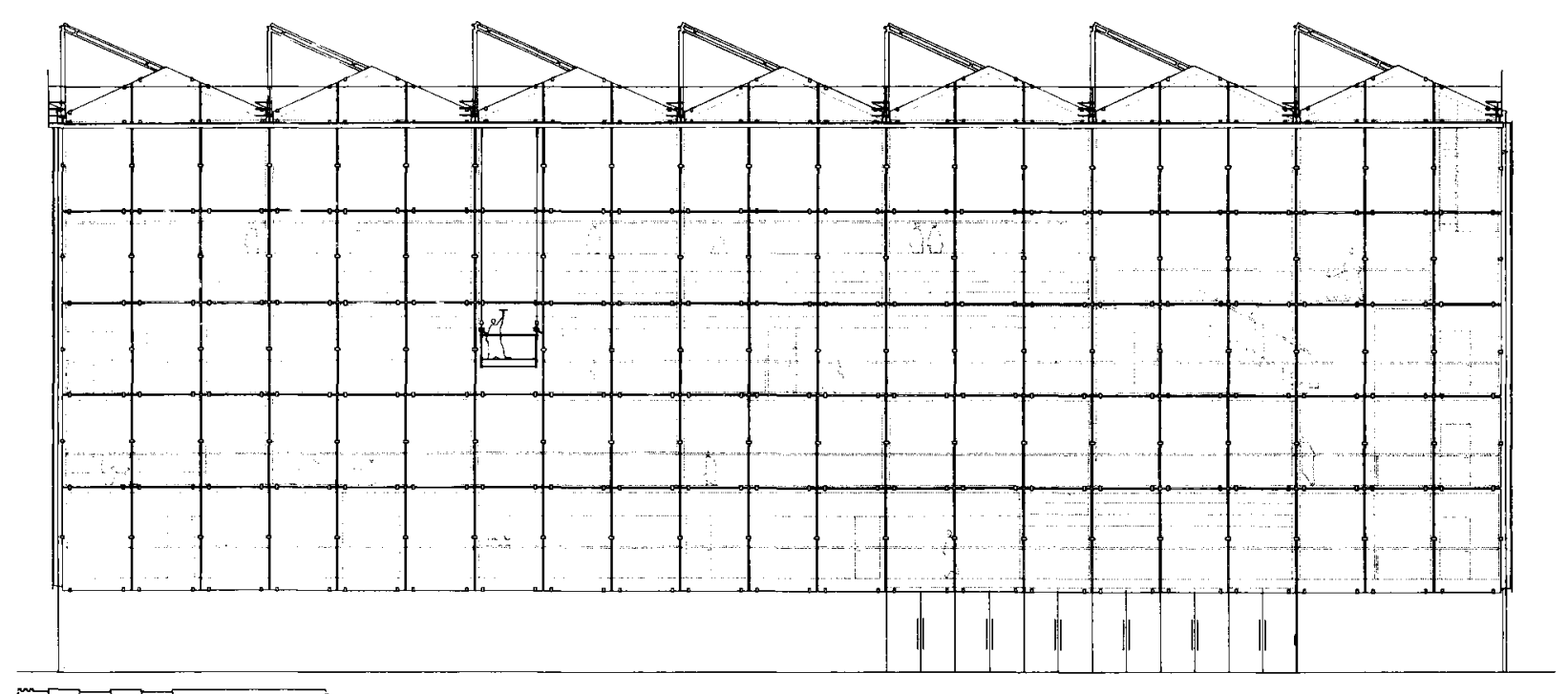


DETALLES DE MURO CORTINA. cotas en $\mathrm{mm}$

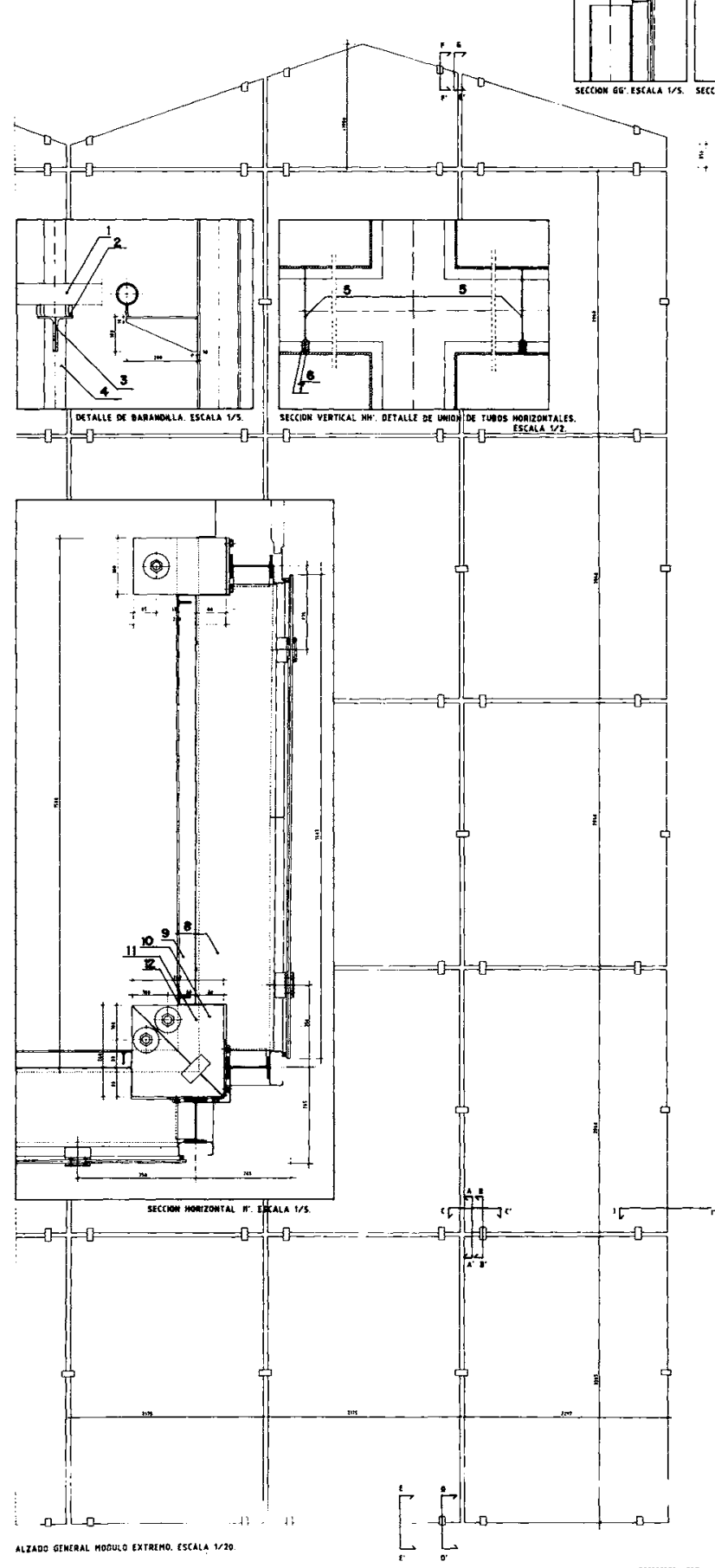

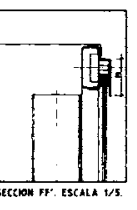
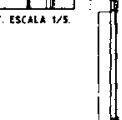
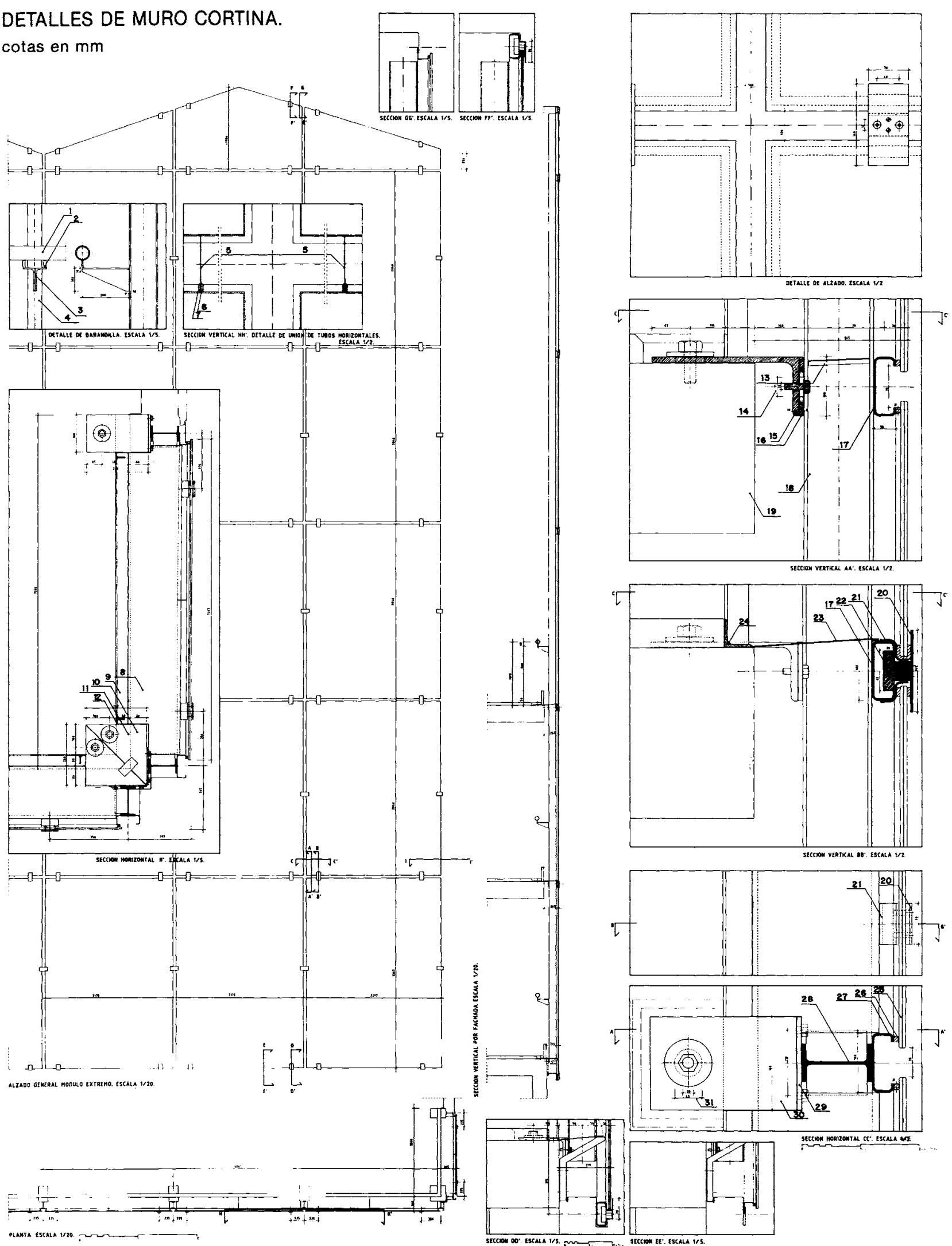

1.- Pasamanos de tubo $\varnothing 60.3$ de acero galvanizado. 2.-Pletina $30 \times 10 \times 6$. 3.-Angular $100 \times 50 \times 64 .-1 P E 120$ del muro cortina. 5.- Junta en el centro de cada módulo. 6.-Pletina de $3 \mathrm{~mm}$ de espesor. 7.- Tapajuntas. 8.-Pieza de remate de muro cortina con forjado. 9.-Rodapié. 10.-Angular de sujección al forjado. 11.-Pletina de unión de los angulares. 12.-Angular soporte de rodapié. 13. - Solapa de cierre de remate en montante. 14. -Tolerancia en mm para movimiento vertical. 15. -Angular $250 \times 100 \times 10$ (perfil compuesto). 16.-Pletina de $3 \mathrm{~mm}$ de espesor. 17.-Tubo horizontal abierto de $3 \mathrm{~mm}$ de espesor. 18.-Perfil IPE 120. 19.-Forjado. 20.- Pieza exterior de presión de acero inoxidable de $4 \mathrm{~mm}$ de espesor. 21.-Refuerzo de tubo horizontal de $3 \mathrm{~mm}$ de espesor. 22. - Pieza interior de presión de acero galvanizado. 23. - Pieza de remate de muro cortina con forjado abatible $N$ de chapa de acero galvanizado. 24.-Angular de 40,5 para remate y base del rodapié. 25.-Vidrio laminado de dos capas de espesor (total 12 mm). 26. - Banda continua de neopreno de $10 \mathrm{~mm}$ de espesor. 27. - Tubo abierto de acero para pintar de $3 \mathrm{~mm}$ de espesor. 28 .- Perfil IPE 120. 29.-Pletina de $6 \mathrm{~mm}$ de espesor. 30.-Angular $250 \times 100 \times 10$. 31.-Tolerancia en mm para movimiento horizontal. 


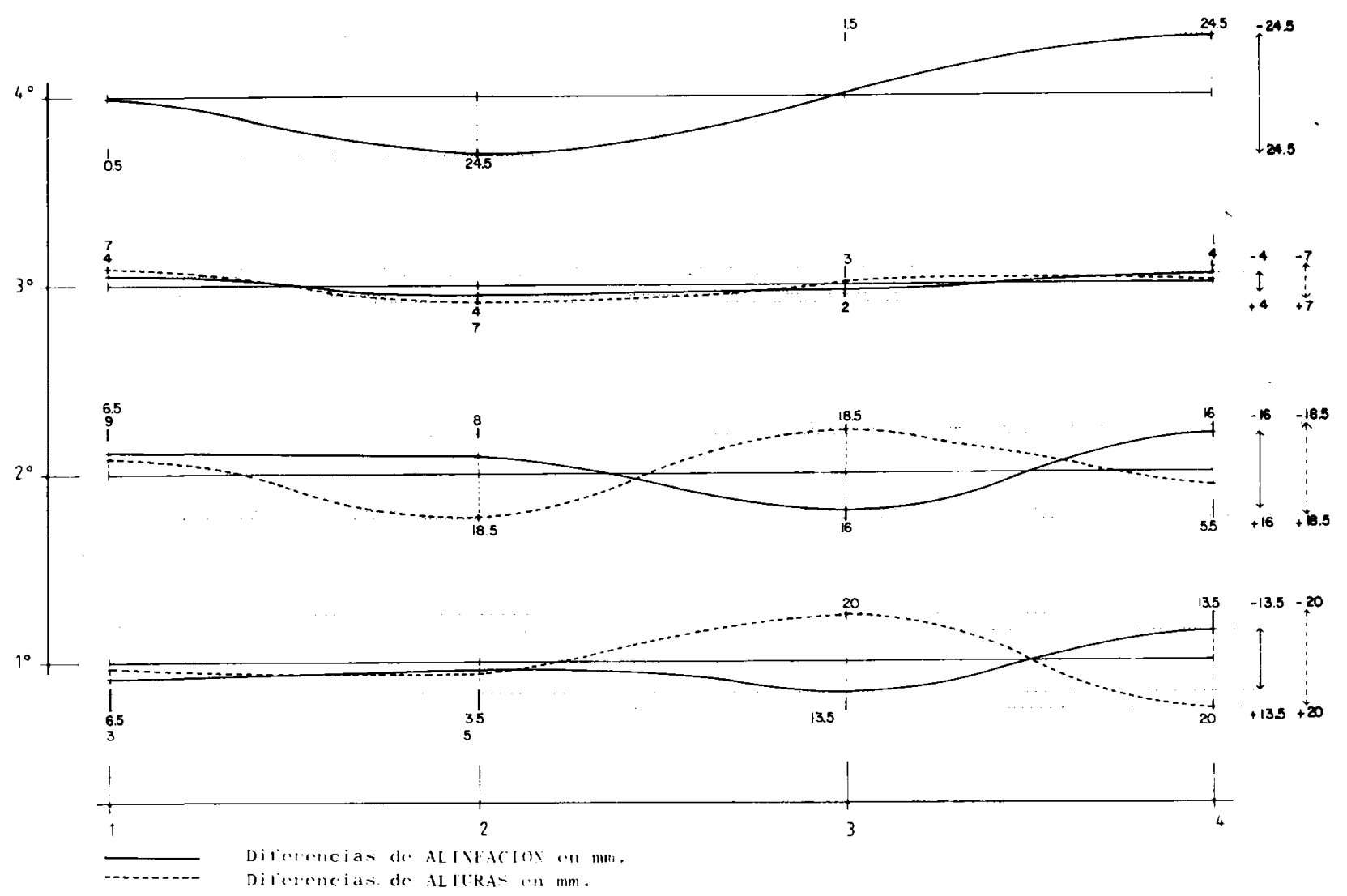

Limites de tolerancias de alturas y alineaciones de testa de forjados: Tolerancia máxima en alineación $+24,5 \mathrm{~mm}$ Tolerancia máxima en altura $+20 \mathrm{~mm}$.

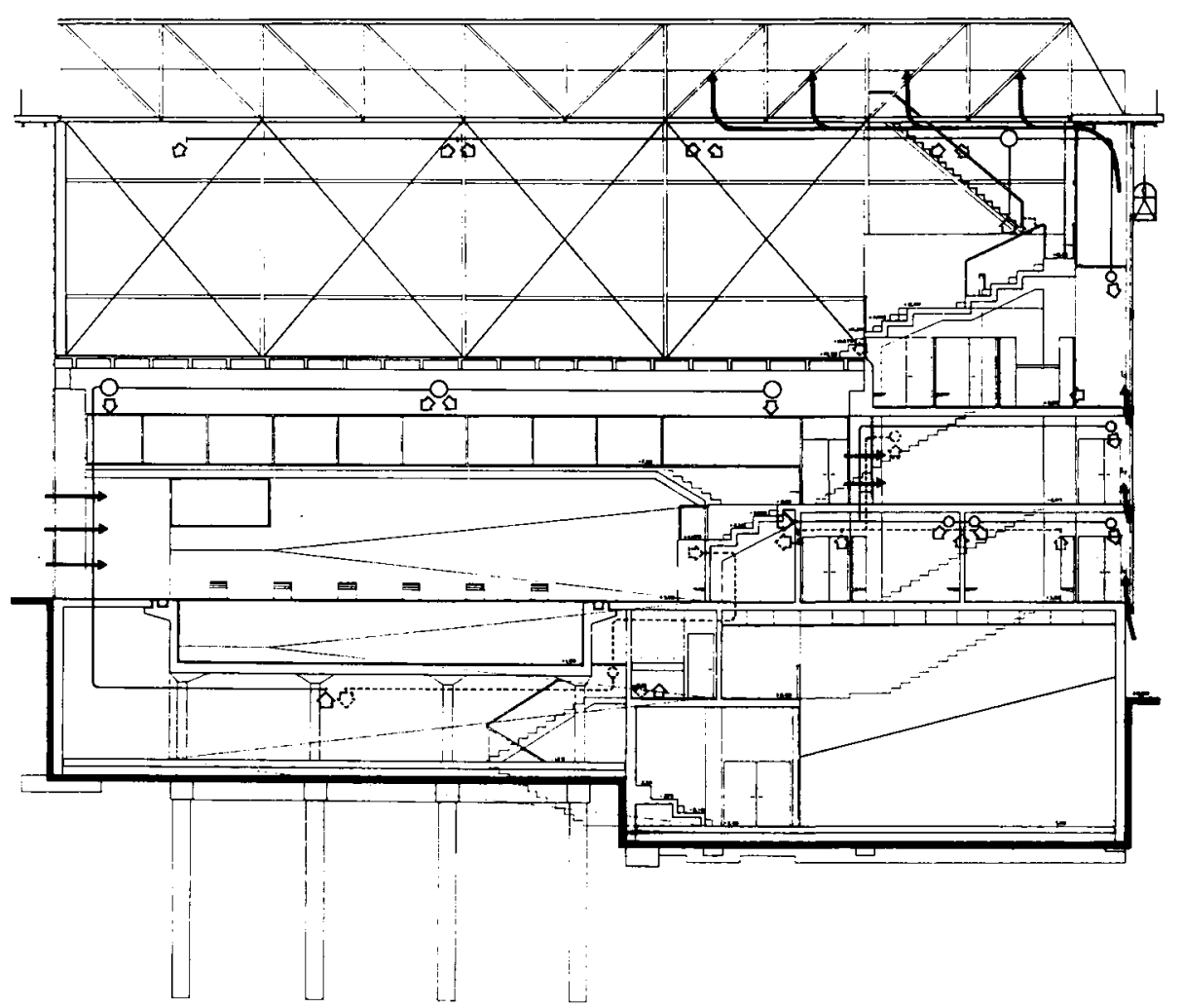

Esquemas de climatización natural a través de la cubierta practicable y de las compuertas de forjado. Esquemas de climatiza. ción artificial en piscina y polideportivo. 
No podemos olvidar tampoco que la historia ha dejado soluciones de arquitectura flexible frente a distin. tas exigencias exteriores, que estarian a medio camino entre la situación actual de control completo del edificio y la inicial de control local del mismo. Hay que combatir la idea romántica de la validez total de las soluciones tradicionales. El progreso de la tecnología y la difusión de los medios mecánicos y de control completan, ampliamente, las posibilidades de la construcción tradicional.
Es preciso investigar y estudiar a rondo estas opciones dejando los estudios románticos sobre tecnologias pobres que ocupan, a un costo incalculable, nuestro tiempo y dinero. Las sociedades desarrolladas se caracterizan por su capacidad para proporcionar más confort, y el futuro o el presente son ya suficientemente significativos como para comprender la imposibilidad de una marcha atrás.

Madrid, febrero 1988

\section{Datos del proyecto}

Proyecto y dirección de obra:

Arquitecto: Salvador Pérez Arroyo

Ingeniero de Caminos: Javier Henche Blanco

Empresa Constructora: Auxini

Muro cortina: $\quad$ Guijarro e Hijos. Monoplex

\section{publicación del IETcc / CSIC}

\section{ACUEDUCTOS ROMANOS EN ESPAÑA \\ Carlos Fernández Casado}

Prof. Dr. Ing. de Caminos, Canales y Puertos

Esta publicación se compone de una serie de articulos, publicados en la Revista "Informes de la Construcción", en los cuales se hace un análisis de los acueductos romanos que existen en Espana y el balance de las condiclones de conservación en que se encuentra cada uno de ellos, incluyendo referencias históricas y literarias. Se ha ilustrado con la reproducción de la valiosa documentación gráfica que posee el prestigioso autor.

Un volumen encuadernado en couché, a dos colores, de $21 \times 27$ centímetros, compuesto de 238 páginas, numerosos grabados, dibujos, fotos en blanco y negro y figuras de linea.

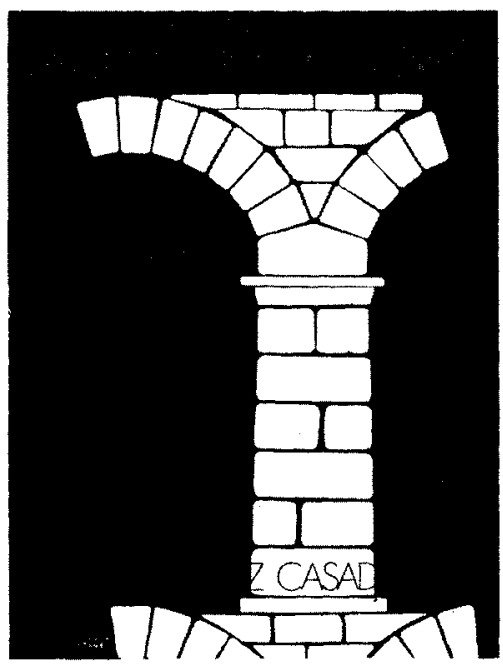

Precio: España, 1.500 plas., 21 \$ USA. 